\title{
Relationship between Job satisfactions with Job burnout among Faculty members of Kurdistan University of Medical Sciences
}

\author{
Research Article
}

\section{Modabber Arasteh ${ }^{1}$, Seyedeh Asrin Seyedoshohadaei ${ }^{2 *}$}

1. Associate Professor of Psychiatry, Department of Psychiatry, Kurdistan University of Medical Sciences, Sanandaj, Iran.

2. Assistant Professor of Psychiatry, Kurdistan University of Medical Sciences, Sanandaj, Iran.

\begin{abstract}
Background and Purpose: Job satisfaction is a positive or positive state, which is the result of an individual job experience assessment. This study was designed to investigate the relationship between job satisfaction and burnout in faculty members of Kurdistan University of Medical Sciences.

Materials and Methods: This cross-sectional study was conducted on 122 faculty members working in Kurdistan University of Medical Sciences in 2010. In order to collect job satisfaction information, a questionnaire with reliability and reliability (Cronbach's alpha, 95\%) and a standardized multivariate questionnaire were used to collect the burnout information from Maslach Burnout Inventory (MBI) to assess the degree of burnout in four The domain of emotional exhaustion, personal performance, personality deprivation and conflict were used.

Findings: The majority of the faculty members were male $(70.5 \%)$, in the medical school $(73.8 \%)$, with a seniority of 10-6 (35\%), and ranked associate professors $(54.1 \%)$. The frequency of burnout was estimated at $73.8 \%$ and $61.5 \%$, respectively. Only $20.5 \%$ of faculty members had job dissatisfaction. There was no statistically significant relationship between age and sex variables, years of service, academic rank, educational groups and salary with job satisfaction and burnout ( $\mathrm{p} \geq 0.05)$.

Conclusion: In general, it can be said that the faculty members of the university have a good job satisfaction, and their job burnout is also acceptable. By examining the causes of dissatisfaction and the factors that contribute to the burnout of the faculty members, Promoted.
\end{abstract}

Keywords: . Job satisfactions, Job burnout, Faculty of members.

\section{Introduction}

The efficiency and effectiveness of the organization depends on the efficiency and effectiveness of the organization's human resources. One of the effective factors in employee productivity is job satisfaction. Various studies on job satisfaction show that organizational, environmental, personal factors and the nature of work are related to job satisfaction (1). Organizationally, a high level of job satisfaction reflects a desirable organizational climate that can increase employees' motivation and productivity (2). Job satisfaction is a situation in which employees enjoy their work in the workplace. He believes that when a staff member has a high level of job satisfaction, this means that he has a positive attitude to his job. Job Satisfaction is a multidimensional instrument of staff, such as satisfaction with job monitoring, satisfaction with payments, working environment conditions, satisfaction with evaluations, satisfaction with individual skills development programs and the like (3). Manpower is

\section{*Corresponding Author:}

\section{Seyedeh Asrin Seyedoshohadaei}

Assistant Professor of Psychiatry,

Kurdistan University of Medical Sciences,

Sanandaj, Iran.

Email: asrin1360@yahoo.co.uk the most important capital of organizations, and the better this quality of capital is, the greater the likelihood of success, survival and promotion of the organization, so much effort should be made on improving the quality of human resources, because it is also in the interest of The organization and in the interests of those who are ready to go beyond the duties assigned. The existence of such a force in the organization, coupled with rising levels of performance, and the reduction of absenteeism, delay, and abandonment of staff, and providing the organization with an appropriate profile in the community, provides a platform for the growth and development of the organization. In contrast, the human resources with a sense of loyalty and a low organizational commitment and a desire to leave the organization not only do not move towards achieving organizational goals, it is also effective in creating a culture of neglect of the organization's issues and problems among other colleagues.

The term job burnout is a term that was introduced for the first time by Freud Burger in the early $1970 \mathrm{~s}$. He is the one who used the term "burnout" with its contemporary theme. He regards it as a form of physical fatigue and emotional frustration, which is due to selflessness and little effort of the individual, in the way of life or career communication. Frederberg (1979) believed that burnout could have a negative effect on the quality of staff productivity and service (4). Job burnout is a kind of individual response to interpersonal 
stressors in the workplace, in which contact over the capacity of the job manager with others leads to changes in attitudes and behaviors toward them (5). Job burnout is a form of physical, emotional and mental fatigue that is caused by frequent and frequent emotional exertion due to long-term exposure to clients (6). Occupational burnout a psychological indicator of emotional exhaustion, depersonalization, and personality deprivation. Emotional exhaustion is quite similar to that of psychological stress, and it's a feeling of being under pressure and loss of emotional resources in an individual. The depersonalization of the person is a negative and rebellious response to those who are usually recipients of service from the individual and refer to the negative perception of the individual of his client. Reducing the sense of adequacy of a person is a diminution of sense of competence in performing a personal task and is a negative self-assessment of work (7). Today, burnout is a product of long-term stress in the workplace and one of the main factors in reducing the efficiency and loss of staffing, which is a problem in all health systems (8). Jackson and Maslach (9) consider job burnout as having three components of emotional exhaustion, personality depersonalization, personality decline, and lack of individual success by reducing personal satisfaction. Organizational factors affecting burnout are: high workload, low labor control, low remuneration, lack of social communication, workplace discrimination, and the contradiction between individual values and environmental values (7). The most significant effect is the burnout, performance drop and the quality of service delivery. These people experience severe emotional exhaustion and exhaustion due to longterm emotional and physical responses to work and stressors in the workplace, and are characterized by excessive absenteeism, excessive use of suffering, a tendency to quit the job and reduce productivity (10).

Job burnout reduces the quality of staffing services and can be considered a factor for lack of accountability, absenteeism and weakening of morale (6). Job burnout is a work-related mental-related injury that causes emotional problems, a reduction in individual success, and personality disorientation (11). Imbalance between job demands and job skills, the difference between resources, expectations and realities of employment and job stress as a major factor in burnout are considered (12), and human resources should also be considered as the most important asset and success factor of the organization (13) Considering that the people who work in the organization play an important role in organizational performance, It seems that the positive emotions and attitudes of individuals in relation to their organization and their jobs can solve many problems and problems in the organization (14) Job Satisfaction consists of a set of feelings and beliefs that people have about their current occupations and is one of the important factors in job success, a factor that increases the efficiency and also the sense of individual satisfaction (15). Dissatisfied people from their work and organization have a good background for mental and physical illnesses and work-related stress is a factor in many of the physical illnesses of the staff. The dissatisfaction with the teacher makes him do his best in the process of education. The dimensions of job satisfaction include: satisfaction with the nature of work, satisfaction with the supervisor, satisfaction with colleagues, satisfaction with promotion, satisfaction of rights and satisfaction with the conditions of the work environment. Commitment is a psychological state that provides some kind of desire, need, and commitment to work in an organization. Job Satisfaction in health and health organizations is one of the important pillars of the promotion, care and treatment of human beings and is of particular importance. Job dissatisfaction among staff not only impedes the quality of the work of the client, but also overestimates the quality of health care (16).

Job burnout is a mental state that includes a set of symptoms such as physical exhaustion, emotional exhaustion and lack of motivation (17). Barry Farber, a burnout specialist, also describes burnout as: job burnout is a lack of coordination between expectations and rewards (18). Job burnout leads to work absenteeism, reduced work incentives, mental disorders, cardiovascular disorders, and even in acute cases of hospitalization (17). Regarding the negative effects of burnout, this syndrome can affect job functions such as job quality, general health and job satisfaction. Boosari (19) showed that burnout has an adverse effect on general health of nurses. Saberi et al (20) also showed that there is a significant relationship between general health and occupational burnout of judges in Tehran. Although several studies have been conducted on job satisfaction, occupational burnout in different occupations, the research has less to do with the interaction between job satisfaction and job burnout on the one hand and on general health. Therefore, considering the importance of the issue and the lack of study in this regard at the university, the recognition of job satisfaction members of the faculty are one of the necessities and they can be used to identify their dissatisfactions in their programs to improve the quality of the performance of professors.

\section{Research Methodology}

This study is a cross-sectional (descriptive and analytical) method. The statistical population of the study consisted of 135 faculty members of Kurdistan University of Medical Sciences who were teaching in 2010. Sample size is equal to the statistical population and the census sampling method, which finally completed 122 questionnaires, and other members did not attend the study because of the mission of traveling abroad or not. A questionnaire used by Safi et al (16) was used to collect Shannon satisfaction data. The Cronbach's alpha in this study was $0.95 \%$. The questionnaire consists of two parts: in the first part, the faculty members' specifications and in the second part of the questionnaire, 45 items were allocated as follows: the first five items related to salaries and benefits, 6 items related to promotion opportunities, 14 items related to the method Career and Communication, 7 related to occupational safety, 7 related to physical conditions and work environment, and 6 related to the nature of work. The Likert scale is used as 0 for non- 
satisfaction of up to 5 for complete satisfaction. The instrument for collecting information on occupational burnout was a standard part of the questionnaire, which included personal characteristics and the Masiach Burnout Inventory (MBI) scale to assess the degree of burnout in four areas of emotional exhaustion, personal performance, personality disorder, and conflict, which was studied by Munir et al (21) in a similar study in Isfahan. The questionnaires were delivered by the moderator to the director of the educational departments or the faculty member at the faculties and educational hospitals. After completing the questionnaires and checking how the questionnaire was completed, they were returned. The completed questionnaires were entered into the computer using the software of the SPSS16 and descriptive statistics (absolute, relative and mean), and chi square test and Fisher exact test.

\section{Research Findings}

In the table (1), the research sample is shown separately based on demographic factors.

Table 1: Frequency distribution of demographic factors in faculty members

\begin{tabular}{|c|c|c|}
\hline \multirow{2}{*}{ Sex } & Man & 86 \\
\cline { 2 - 3 } Academic Degree & Female & 36 \\
\cline { 2 - 3 } & Instructor & 52 \\
\hline \multirow{4}{*}{ College of Service } & $\begin{array}{c}\text { Assistant } \\
\text { Professor }\end{array}$ & 66 \\
\cline { 2 - 3 } & $\begin{array}{c}\text { Associate } \\
\text { Professor }\end{array}$ & 4 \\
\cline { 2 - 3 } & Nursing School & 21 \\
\cline { 2 - 3 } & Faculty of health & 10 \\
\cline { 2 - 3 } Years of service & $\begin{array}{c}\text { Faculty of Para } \\
\text { medicine }\end{array}$ & 1 \\
\cline { 2 - 3 } & Medical School & 90 \\
\hline & $\mathbf{1 - 5}$ & 24 \\
\cline { 2 - 3 } & $\mathbf{6 - 1 0}$ & 37 \\
\cline { 2 - 3 } & $\mathbf{1 1 - 1 5}$ & 23 \\
\cline { 2 - 3 } & $\mathbf{1 6 - 2 0}$ & 22 \\
\hline \multirow{3}{*}{$\mathbf{2 1}$ years and older } & 16 \\
\hline
\end{tabular}

In table (2), the status of occupational stress and burnout is studied at various levels.

Table 2: General status of job satisfaction and burnout in faculty members

\begin{tabular}{|c|c|c|c|}
\hline \multicolumn{2}{|c|}{ Variables } & Number & $\%$ \\
\hline \multirow{4}{*}{$\begin{array}{c}\text { Job } \\
\text { Satisfaction }\end{array}$} & $\begin{array}{c}\text { Somewhat } \\
\text { dissatisfied }\end{array}$ & 25 & $20.5 \%$ \\
\cline { 2 - 4 } & $\begin{array}{c}\text { Somewhat } \\
\text { satisfied }\end{array}$ & 72 & $59 \%$ \\
\cline { 2 - 4 } & Satisfied & 23 & $19 \%$ \\
\cline { 2 - 4 } & Very satisfied & 2 & $1.5 \%$ \\
\hline \multirow{2}{*}{$\begin{array}{c}\text { Job } \\
\text { Burnout }\end{array}$} & Mild & 90 & $73.8 \%$ \\
\cline { 2 - 4 } & Medium & 32 & $26.2 \%$ \\
\hline
\end{tabular}

The overall burnout rate for most faculty members is low (26.2\%), And about $21 \%$ faculty members are happy with their job.

\section{Discussion and conclusion}

Increasing job satisfaction improves various aspects of job attitude, such as organizational commitment, occupational attachment, organizational climate and job burnout (22).

In general, the faculty members of Kurdistan University of Medical Sciences are satisfied with their job and burnout is relatively low, which is consistent with studies by Safi et al (22) and Teymouri et al (23), but with other issues such as Foroughi et al (24) does not match.

The satisfaction of the faculty members of the university varies from one area of activity to the other. Since the satisfaction of the work environment is very good, the salary, the level of supervision and communication are good, but from the scope of promotion opportunities, job security and physical condition, their satisfaction is moderate is. Which is partly consistent with the results of Teymouri et al (23). Examples of such issues as income level, management, and lack of study opportunities have been the cause of their dissatisfaction. With regard to the above, it seems that the teaching and training faculty members are pleased and care about.

The satisfaction of the faculty members with good supervision and communication was inadequate in the study of Foroughi et al (24) in Kermanshah, which satisfaction with management and supervision was moderate (23). Appropriate and friendly approach and creating an environment that is far from tension by the authorities plays an important role in job satisfaction. Also, friendly and intimate relationships with supervisors and managers and officials play a significant role in personnel job satisfaction.

In this study, the relatively low satisfaction of faculty members with job security is not consistent with the results of other similar studies $(24,23)$. Since job security is the main tool in the organization's commitment to the individual and the sense of security of the apple, the support and stability of the individual is effective in providing the service, so finding the cause seems important. On the other hand, according to the information provided by faculty members, no faculty member has been dismissed by the university. This lack of job security is likely to include issues such as the annual assessment of board members to promote selection.

In this study, the burnout of teachers in the area of emotional exhaustion and depersonalization was low and in the performance of the sheikh at moderate level and in the extent of conflict was high, and in general, the degree of burnout in $73.8 \%$ of the faculty members was low, which is The findings are in line with the results of the study by Munir et al (21) that examine the burnout of faculty members in universities. In their study, they showed that personal performance was excessively moderate in terms of frequency and severity and was moderate in terms of emotional exhaustion, depersonalization and conflict. Based on the findings, 
this study did not show a significant statistical correlation between the burnout of faculty members with age and sex, faculty of service, academic rank, years of service, and salary, which is consistent with the results of Munir et al (21).

In general, it can be said that the faculty members have a good job satisfaction. Also, burnout can improve the status quo. They are also acceptable. The status quo can be improved by examining the causes of dissatisfaction and the factors that contribute to the burnout of faculty members.

\section{Reference}

1. Tabatabaei, Shahnaz, Simin Hosseinianb, Bahman Gharanjiki, General health's, stress associated to the work and job satisfaction of Hormozgan Cement Factory employees in Iran, Procedia - Social and Behavioral Sciences 30 (2011) 1897 - 1901.

2. Tajvar M, M Arab and V Khodabakhsh Nejad, Personnel job satisfaction in Tehran Koodakan kids medical center, Journal of Health Administration, 2006 (Issue 23).

3. Brunetto, Y., \& Farr-Wharton, R. (2002). Using social identity theory to explain the job satisfaction of public sector employees. International journal of public sector management, 15(7), 534-551.

4. Rass, Rendaal \& Eltmaayer, Elizaabet. (1995). Job Stress; Translate G. Khwaja Poor.Tehran: Baaztaab.

5. Saaetchi, M.(2007). Productivity Psychology. Tehran: Institute of Print Edition.

6. Bordbar, A.(2008).Burnout and ways to counter them. Journal of eighteen. 14,(-), pp:12-74.

7. Maslach C, Job Burnout. Annual Review of Psychology, 2001; 52:397-422.

8. Fredenberger HJ, Staff Burnout. J Soc Iss, 1974;30:159-165.

9. Jackson D \& Maslach C, Burnout: A Social Psychological Analysis. Hum Behav, 1976; 5:16-22

10. Polikandrioti M. Burnout syndrome. Health Science Journal, 2009;3:195-6.

11. Nazem,F \& Alizadeh Ranjbar, T. (2014). The relationship between empowerment and quality of working life With burnout Among employees of Education. Quarterly Journal of New Approaches in Educational Administration, Vol 5,No (2), pp: 91108.

12. Mohajer, Mehdi. ( 2003 ). The relationship between personality traits, Career history with burnout among teachers Tabriz city. graduate thesis. University of Tabriz.

13. Mohammadian, Yadallah, Mahdavi, Moosa \& Hoseyni nezhad, aeazam. (2010). Study of job satisfaction and organizational commitment in a military university. Journal of Military Psychology. 1, (27), pp:25-36.
14. Zamini,S . ,Hoseyninasab, D. ,Zamini,S \& Zareaei, P .(2011). Examine the relationship between organizational culture, job satisfaction and burnout among staff of the University of Tabriz. Journal of Occupational Health. 8 ,( 1 ), pp: 30.

15. Sabzipoor, Amir, Reshnoodi, Behzad, Amiri, Somayeh \& Karam Elahi, Ali. (2014). The relationship between the type of personality introversion-extraversion with Job Satisfaction and Organizational Commitment of Teachers. Quarterly Journal of New Approaches in Educational Administration, Vol.5, (2), pp: 181-194.

16. Safi.Mohammad-Hossein, Mohamadi. Farima \& Kolahi. Ali-Asghar, (2015), The Relationship between Job Satisfaction with Burnout and Conflict Management Styles in Employees, Journal of Social Determinants of Health Research Center of Community Health; Vol. 2, No. 4.

17. Hakanen, Jari J. Wilmar B. Schaufeli, Do burnouts and work engagement predict depressive symptoms and life satisfaction? A three-wave seven-year prospective study, Journal of Affective Disorders 12 (4), (2012), 312-329.

18. Lydia J. Goutas, Burnout, TheWrite Stuffs, The Journal of the European Medical Writers Association, Vol. 17, No. 3, 2008.

19. Payami Boosari M, Burnout syndrome in nurse, working at educational hospital Zanjan, Iran Journal of Nursing, 2003(Issue 33).

20. Saberi SM, et al, The relation between job burnout and general health of judge and prosecutors working in courts of Tehran. HBI Journals. 2008; 14 (2) :92-98

21. Munir F,Nielsen $\mathrm{K}$, garde $\mathrm{AH}$, Albertsen K,Carneiro IG.Mediating the effects of work-life confict between transform care workers job satisfaction and psychological wellbeing. Journal Of Nursing Management.2011;20(4):512-21.

22. Safi M H, Falahi Khoshknab M, Russel M, Rahgozar M. The Effective Individual, Environmental and Occupational Factors of Job Satisfaction amongst the Academic Staff of University of Social Welfare and Rehabilitation Sciences. Strides Dev Med Educ. 2010; 7 (1) :3443.

23. Teymouri M, Tootoonchi M, Salehi M, Hassanzadeh A. Job Satisfaction among Faculty Members of Isfahan University of Medical Sciences. Iranian Journal of Medical Education. 2008; 7 (2) :227-237.

24. Foroughi F, Kharrazi H, Iranfar S, Rezaei M. Job Satisfaction and its Affecting Factors from the Viewpoints of Faculty Members of Kermanshah University of Medical Sciences. Iranian Journal of Medical Education. 2008; 7 (2) :335-342. 